\title{
Consultas de enfermagem realizadas em uma unidade de saúde da família da cidade de Atibaia - SP
}

\author{
Nursing consultations held in a unit of the family health \\ of the city of Atibaia, SP - Brozil
}

\author{
Tamara de Moraes Cillo'; Rogério Barbosa de Deus²; Anderson Sena Barnabé3; \\ Renato Ribeiro Nogueira Ferraz ${ }^{4}$ \\ Enfermeira - UAM/SP. Especialista em Saúde Coletiva com ênfase em Programa de Saúde da Família - Uninove. São Paulo, SP - Brasil \\ ${ }^{2}$ Médico - UFMG. Nefrologista pelo Hospital das Clínicas - UFMG. Mestre e Doutor em Nefrologia - Unifesp. São Paulo, SP - Brasil \\ ${ }^{3}$ Biólogo, Mestre e Doutor em Saúde Pública - Faculdade de Saúde Pública/USP, Docente da Disciplina de Epidemiologia do Curso \\ de Pós Graduação em Saúde; Pública - Uninove/SP, pesquisador do NESCOF (Núcleo de Estudos em Saúde da Comunidade e da \\ Família) - Uninove. São Paulo, SP - Brasil \\ ${ }^{4}$ Bacharel e Licenciado em Ciências Biológicas - UniABC/SP. Mestre e Doutor em Nefrologia - Ciências Básicas -UNIFESP/SP. Docente \\ da Disciplina de Metodologia do Ensino e da Pesquisa, do Curso de Pós-graduação em Saúde Coletiva com Ênfase em Programa de \\ Saúde da Família (PSF) - Uninove. São Paulo, SP - Brasil
}

\section{Endereço para correspondência \\ Renato Ribeiro Nogueira Ferraz \\ Av. Pedro Mendes, 872 - Parque Selecta \\ 09791-530 - SBCampo, SP [Brasil]}

renato@nefro.epm.br

\section{Resumo}

Objetivos: Objetivou-se quantificar e categorizar as consultas de enfermagem realizadas em uma unidade de Saúde da Família da cidade de Atibaia - SP. Método: A amostra foi composta por 78 pacientes, sendo 31 do sexo feminino, e 47 , do masculino. Os dados foram agrupados de acordo com o tipo de consulta de enfermagem e categorizados da seguinte maneira: puericultura, pré-natal, papanicolau, hipertensão, diabetes e acolhimento. Resultados: Os resultados revelaram que a hipertensão arterial sistêmica e o diabetes mellitus são as principais doenças de base cujas complicações são comumente observadas nas consultas de enfermagem, sendo essas de extrema valia para um melhor prognóstico do paciente, quando conduzidas adequadamente. Conclusão: A eficácia e o profissionalismo do enfermeiro na execução do seu processo de trabalho estimula o surgimento de um importante vínculo com o paciente, contribuindo para a sistematização e otimização dos atendimentos, reduzindo custos e dispêndio de tempo.

Descritores: Atendimentos; Consulta de enfermagem; Prevalência; Unidade básica de saúde.

\begin{abstract}
Objective: This study aimed to quantify and categorize the nursing consultations carried out in a Family Health unit of Atibaia - SP, Brazil. Methods: The sample consisted of 78 patients, 31 females and 47 males. The data were grouped according to the type of nursing consultation and categorized as follows: child care, prenatal care, papanicolau, hypertension, diabetes and host. Results: The data revealed that hypertension and diabetes mellitus are the main underlying diseases whose complications are commonly seen at nursing consultation, being extremely important for a better prognosis of the patient, when conducted properly. Conclusions: The effectiveness and professionalism of the nurse in implementing the process of work encourages the emergence of an important relationship with the patient, contributing to the systematization and optimization of care, reducing costs and time.
\end{abstract}

Key words: Basic health unit; Care; Nursing consultation; Prevalence. 


\section{Introdução}

A realização das consultas de enfermagem faz parte do cotidiano profissional dos enfermeiros. Para muitos, além de identificar os problemas de saúde do cliente, esse tipo de consulta proporciona a criação de um importante vínculo entre o profissional e o usuário do sistema de saúde. Os enfermeiros estão corretos em afirmar que, durante uma consulta individual, não apenas os sinais e sintomas biológicos do cliente devem ser observados, mas também as condições sociais e a maneira como esse enfrenta seus problemas, são informações que devem ser consideradas ${ }^{1}$.

O Sistema Único de Saúde (SUS) foi criado com o firme propósito de alterar a situação de desigualdade na assistência à saúde da população, universalizando o acesso ao atendimento público e tornando-o obrigatório e gratuito a qualquer pessoa. É um sistema público, ou seja, destinado a toda população, e financiado com recursos arrecadados por meio de impostos pagos por todos os indivíduos, usuários ou não do serviço².

A partir da existência do SUS, foram implementados vários modelos assistenciais, como o Programa de Saúde da Família (PSF) que, segundo Santos ${ }^{3}$ "[...] representa uma nova estratégia de política de focalização dos serviços assistenciais de saúde e que surpreendentemente vem causando uma verdadeira evolução no campo da saúde pública".

Em 1998, o Ministério da Saúde (MS) oficializou o Programa de Saúde da Família por meio da Lei no. 2.177 de 30-12-1998 e do decreto $\mathrm{n}^{\circ}$ 2.043 de 23-2-1999, que estabelecem as gestões do programa "[...] a filosofia do PSF baseia-se na doutrina do SUS com intuito de buscar a reorganização da prática assistencial em novas bases e critérios, tendo como objetivo o atendimento ao indivíduo no seu contexto familiar"1.

De acordo com Santos ${ }^{4}$, o PSF apresenta-se como um programa relevante por trazer na fundamentação de seu discurso novas perspectivas técnicas e ideológicas de reorganização e de intervenção do modelo assistencial vigente no país, contrapondo-se nas raízes com as práticas, tradi- cionais dessa assistência. A referida autora também chama a atenção para a significativa abertura do mercado de trabalho nesse setor e nele se inserindo uma "nova" categoria profissional a do agente comunitário de saúde (ACS).

Tomando-se como exemplo a cidade de Atibaia (SP), a implementação do programa, no ano de 2001, iniciou-se com a formação de 11 equipes. Atualmente, esse município conta com 19 equipes em atuação. As equipes mínimas do programa são constituídas por um médico, um enfermeiro e quatro agentes de saúde que tomam sob sua responsabilidade a assistência de aproximadamente 600 famílias.

Na avaliação desses programas, levam-se em consideração especialmente as atividades desenvolvidas pelo enfermeiro, que tem como atribuições realizar cuidados diretos de enfermagem, fazer a indicação para a continuidade da assistência prestada, realizar consulta de enfermagem, solicitar exames complementares, prescrever/transcrever medicações conforme protocolos estabelecidos pelo Ministério da Saúde e as disposições legais da profissão, planejar, gerenciar, coordenar, executar e avaliar a Unidade de Saúde da Família (USF).

A consulta de enfermagem foi reconhecida como uma rotina exclusiva do enfermeiro, no Brasil, em 1986, com a aprovação da nova legislação do exercício profissional, não sendo, portanto, delegável a outros.

Seria interessante que as USFs realizassem um levantamento periódico do número de consultas de enfermagem realizadas por período, com o intuito de conhecer a população atendida e criar um programa de sistematização do atendimento para melhor amparar seus usuários, minimizando custos e tempo de espera por atendimento, e contribuindo assim para o sucesso do programa.

\section{Objetivo}

Quantificar as consultas de enfermagem realizadas em uma unidade de saúde da família na cidade de Atibaia (SP). 


\section{Método}

Trata-se de um estudo prospectivo, descritivo e de natureza quantitativa realizado de 20 de julho a 9 de agosto de 2009, em uma unidade de saúde da família (USF), localizada no município de Atibaia (SP). No decorrer do período citado, dados referentes ao sexo, idade e motivo de procura pelo serviço foram coletados de todos os pacientes atendidos pela USF em questão, que autorizaram a utilização de suas informações por meio da assinatura do Termo de Consentimento Livre e Esclarecido. Esses dados foram inseridos em planilha eletrônica, analisados e apresentados pelos seus valores inteiros e percentuais, sem a aplicação de testes estatísticos. Não foi divulgada nenhuma informação que pudesse identificar os pacientes ou a instituição em que este trabalho foi realizado. Esta pesquisa foi registrada no Conselho Nacional de Ética em Pesquisa (CONEP), sob o n ${ }^{\circ} 286388$, estando de acordo com a resolução 196/96 do Conselho Nacional de Saúde quanto aos seus aspectos éticos e legais. O levantamento dos dados e a confecção deste estudo foram aprovados pelo Departamento de Supervisão Técnica da referida USF por meio de documento oficial.

\section{Resultados}

Observou-se que a maioria das consultas de enfermagem se refere a acompanhamentos de pacientes com doenças crônicas, destacandose a hipertensão e o diabetes mellitus, além de acompanhamentos de rotina, por exemplo, puericultura (acompanhamento do desenvolvimento infantil) e papanicolau (prevenção de câncer de colo do útero), somados a uma pequena quantidade de pacientes em acolhimento.

Dos 78 pacientes observados, 31 eram do sexo feminino $(40 \%)$, e 47 , do masculino $(60 \%)$, apresentando em média $37 \pm 16$ anos. Quanto aos motivos de atendimento, $28 \%$ do total da amostra (22 indivíduos) tinham hipertensão arterial, sendo 11 homens (50\%) e 11 mulhe- res (50\%). Do total da amostra, 23\% (18 pacientes) foi composta por indivíduos com diabetes mellitus, 8 mulheres (45\%) e 10 homens (55\%). Dos indivíduos atendidos, 12 (15\%) procuraram a unidade para consulta de acolhimento, sendo 5 mulheres (41\%) e 7 homens (59\%). Do total, 8 crianças $(11 \%)$ foram à Unidade para consulta de Puericultura, 5 meninos (62\%) e 3 meninas (38\%). Para acompanhamento de pré-natal, foram computados 5 atendimentos (6\%), já para a realização do exame de Papanicolau, no período citado, ocorreram 13 (17\%).

\section{Discussão}

O programa de saúde da família tornouse a estratégia prioritária de reformulação do modelo assistencial da atenção básica, estando já implantadas cerca de 10 mil equipes de saúde da família no país 5 . Esse número encontra-se em constante evolução, dado o ritmo de crescimento do total de equipes.

Segundo Campos ${ }^{6}$, o vínculo com os usuários do serviço de saúde amplia a eficácia das ações de saúde e favorece a participação do usuário durante a prestação do serviço. Esse espaço deve ser utilizado para a construção de sujeitos autônomos, tanto profissionais quanto pacientes, pois não há construção de vínculo sem que o usuário seja reconhecido na condição de sujeito, que fala, julga e deseja. Franco ${ }^{7}$, afirma que a relação humanizada da assistência, que promove a acolhida, dá-se sob dois enfoques: o do usuário e o do trabalhador.

A consulta de enfermagem constitui-se como importante atribuição do enfermeiro, pois é a partir dessa que são colhidas informações relevantes sobre o paciente. É o primeiro contato que o indivíduo tem com o profissional de saúde, em que se estabelece o vínculo entre quem cuida e quem necessita de cuidados. Durante este estudo, confirmou-se que a consulta de enfermagem realmente é o principal meio de comunicação entre o enfermeiro e o paciente, que possibilita ao atendido expor seus anseios e desejos ${ }^{8,9}$. 
Para viabilizar um melhor atendimento e até solucionar grande parte das questões que chegam ao conhecimento do enfermeiro, é necessário que essa categoria profissional compreenda a real importância da consulta de enfermagem. Essa consulta é um momento único da relação cuidador-paciente, formando-se por meio dela, como dito, um importante vínculo de confiança que contribui para sanar as necessidades individuais de cada paciente. É no momento dessa consulta que o paciente pode apreender a importância de cuidar-se, constituindo-se como valioso instrumento para o conhecimento da pessoa de forma holística. Ainda, por intermédio da consulta, tem-se a oportunidade de tirar dúvidas a esclarecer conceitos.

Neste trabalho, $28 \%$ da amostra foi constituída por indivíduos com hipertensão arterial, sendo essa uma condição patológica muito comum na atualidade. Muitas pessoas nem desconfiam que possuem níveis pressóricos elevados, já que o organismo acostuma-se com a hipertensão que, contudo, vai comprometendo em silêncio órgãos-alvo como o coração, rins, cérebro e olhos. A hipertensão nada mais é do que o aumento da pressão exercida pelo sangue sobre as artérias, gerada pela contração do ventrículo esquerdo, e que pode ser aferida pela tomada de dois valores: máxima (pressão sistólica), que diz respeito à pressão que o coração faz para bombear o sangue em direção a todo o organismo durante a sístole ventricular esquerda, e a mínima (pressão diastólica), que se refere à acomodação do sangue nos vasos sanguíneos durante a diástole da câmara cardíaca citada ${ }^{10}$.

Neste estudo, 23\% dos pacientes tinham Diabetes mellitus. O Diabetes mellitus é um distúrbio no metabolismo da glicose, a qual, presente em excesso no sangue, passa à urina sem ser usada como um nutriente pelo corpo. Existem, porém, diferenças nas causas e na gravidade desse distúrbio. Por isso, costuma-se falar em diferentes tipos de diabetes, sendo os dois mais comuns o Diabetes Tipo I e o Tipo II. A distinção entre um e outro, nem sempre é fácil ${ }^{10}$.
Dos indivíduos observados, 15\% procuraram a unidade para passar em acolhimento. Segundo Matumoto ${ }^{11}$, acolhimento e vínculo podem ser identificados no encontro do trabalhador com o usuário durante o trabalho vivo em ato que é o trabalho em ação, ou seja, que está em processo de construção. Durante essa atividade, o trabalhador pode ser criativo e autônomo quanto aos instrumentos à sua disposição, dependendo do objetivo que pretenda atingir. Justifica-se analisar acolhimento e vínculo no PSF pela necessidade de conhecer-se o que de fato ocorre durante a realização do trabalho em saúde, bem como apreender a intencionalidade do trabalhador por meio do projeto pensado, antes mesmo de sua execução.

Do total, cerca de $10 \%$ dos pacientes foram à unidade para consulta de puericultura. A puericultura, área da pediatria voltada principalmente para os aspectos de prevenção de promoção da saúde, atua no sentindo de manter a criança saudável para garantir seu pleno desenvolvimento, de modo que atinja a idade adulta sem influências desfavoráveis e problemas trazidos da infância. Suas ações priorizam a saúde em vez da doença. Seus objetivos básicos contemplam a promoção da saúde infantil, prevenção de doenças e educação da criança e de seus familiares, por meio de orientações antecipatórias aos riscos de agravo à saúde, podendo oferecer medidas preventivas mais eficazes. Para ser desenvolvida em sua plenitude, deve-se conhecer e compreender a criança em seu ambiente familiar e social, além de suas relações e interação com o contexto socioeconômico, histórico, político e cultural em que está inserida. Isto se torna fundamental, pois as ações médicas, além de serem dirigidas à criança, refletem-se sobre o seu meio social, a começar pela família ${ }^{5}$.

Por volta de $7 \%$ da amostra deste trabalho realizam consultas de pré-natal - nome dado ao acompanhamento de enfermagem dedicado à mulher e ao bebê durante todo o período gestacional. Neste acompanhamento, o enfermeiro dá instruções à futura mamãe, como cuidados com a alimentação, formas de manter-se confor- 
tável, estimulação do mamilo para um processo de amamentação mais eficaz, uso adequado de polivitamínicos e outras, bem como a necessidade da realização de exames e continuidade do acompanhamento até o fim da gestação. As consultas devem ser iniciadas o quanto antes para que possam ser feitos os exames necessários que vão garantir a saúde da gestante e do bebê, assim como a detecção de alguma doença ou disfunção, se estas existirem. Os exames identificam a gestação normal e a de alto risco, na qual a mãe ou o bebê apresentam alguma doença que possa representar qualquer tipo de risco a ambos ${ }^{12}$.

Por sua vez, $17 \%$ da amostra procuraram a unidade para fazer coleta de Papanicolau. A citologia cervical, ou papanicolaou, é o exame preventivo do câncer do colo uterino. O exame deve ser realizado em todas as mulheres com vida sexual ativa ou não, pelo menos uma vez ao ano. Após três exames anuais consecutivos normais, o teste de Papanicolau pode ser realizado com menor frequência podendo ser, em mulheres de baixo risco, até a cada três anos, de acordo com a análise do médico. Todavia, mulheres com pelo menos um fator de risco para câncer do colo uterino devem continuar se submetendo ao exame anual. Esse exame consiste basicamente na coleta de material do colo uterino com uma espátula especial, sendo esse material colocado em uma lâmina e analisado posteriormente por patologistas. Essa simples prática contribui enormemente para a redução da incidência de casos de câncer de cólo de útero ${ }^{12}$.

\section{Conclusão}

Durante a consulta de enfermagem, o enfermeiro cuida do ser humano como um todo, esclarecendo questões, contribuindo, de certa forma, para um melhor prognóstico. É na consulta de enfermagem que o paciente passa a ter uma visão inovadora sobre as práticas de saúde e sobre como cuidar de seu corpo e mente, tendo uma vida mais saudável e aprendendo conhecimentos essenciais. Nosso estudo reafirma o quanto é importante olhar, ouvir e observar o que os pacientes têm a dizer, visando o sucesso do processo de cuidar.

\section{Referências}

1. Bicca LH, Tavares KO. A atuação da enfermeira no Programa de saúde da Família. Uma breve análise da sua prática assistencial. Nursing. 2006;92(9):632-7.

2. Alves Sobrinho EJ, Capucci PF. Saúde em São Paulo: aspectos da implantação do SUS no período de 20012002. Estudos Avançados. 2003;17(48):209-27.

3. Santos RL. Homens como agentes comunitários de saúde: trabalhando cuidados e vivenciando gênero. Tese [Doutorado em Saúde Pública]. Universidade de São Paulo. São Paulo; 2005.

4. Santos AS. Os modelos técnicos assistenciais e o Sistema Único de Saúde. Nursing. 2006;96(9):823-34.

5. Alves CR, Viana MR, Saúde da família: Cuidando de crianças e adolescentes. Belo Horizonte: Editora Coopmed; 2003.

6. Campos GW. Considerações sobre a arte e a ciência da mudança: revolução das coisas e reforma das pessoas. O caso da saúde. In: Cecília LC. Inventando a mudança na saúde. $2^{\text {a }}$ ed. São Paulo: Hucitec; 1997. p. 113.

7. Franco TB, Merhy EE. O uso de ferramentas analisadoras para apoio ao planejamento dos serviços de saúde: o caso do serviço social do hospital das clínicas da UNICAMP (Campinas SP). Em: Merhy EE. O Trabalho em Saúde: olhando e experienciando o SUS no cotidiano. São Paulo: Hucitec; 2004.

8. Silva MJP. O toque e a distância interpessoal entre enfermeiros e pacientes nas consultas de enfermagem. Rev Esc Enferm. USP. 1991; 25(3):309-18.

9. Silva MG. Consulta de enfermagem no contexto da comunicação interpessoal - A percepção do cliente. Rev Latino-Am Enfermagem. 1998;6(1):27-31.

10. Massironi MMg. Protocolo de tratamento da hipertensão arterial e do diabetes melito tipo II na atenção básica. Prefeitura Municipal de São Paulo. Secretaria Municipal da Saúde. São Paulo, 2008. 108 p.

11. Matumoto S, Mishima SM, Pinto IC. Saúde coletiva: um desafio para a enfermagem. Cad Saúde Pública. 2001;17(1):233-41.

12. BRASIL. Ministério da Saúde. Atenção básica à saúde da mulher. Brasília, DF. 2006. p. 89-150. 
